between MES and skin involvement (mRss) $(\mathrm{p}=0.05)$. On the other hands, FRAX, the major osteoporotic fracture risk, positively correlates with Medsger's kidney disease severity $(p=0.04)$ and Medsger's lung disease severity $(p=0.04)$; in addition, FRAX, for hip fracture risk, seems to correlate significantly with Medsger's lung involvement severity $(p=0.04)$.

Conclusions: This study demonstrates in SSc patients a relationship between clinical disease severity (organ fibrosis/failure) and altered bone microarchitecture (TBS). In addition, skin involvement was found significantly correlated with altered quality of the trabecular bone architecture (TBS) and a significant increase of osteoporotic fracture risk (FRAX) was found correlated with kindey and lung involvement.

References:

[1] Varga J, Abraham D. J Clin Invest. 2007 Mar;117(3):557-67.

[2] Loucks J, Pope JE. Semin Arthritis Rheum. 2005 Feb;34(4):678-82.

[3] Silva BC et al. J Bone Miner Res. 2014 Mar;29(3):518-30.

[4] Medsger TA et al. Clin Exp Rheumatol. 2003;21(3 Suppl 29):S42-6.

[5] Sulli A, et al. Ann Rheum Dis 2008;67:885-7.

[6] Cutolo et al. J Rheumatol 2000; 27:155.60.

Disclosure of Interest: None declared

DOI: 10.1136/annrheumdis-2017-eular.6415

\section{FRI0570 OSTEOPOROSIS AND BREAST CANCER: OUTCOMES AT A SPECIALIZED OSTEOPOROSIS CLINIC FOLLOWING A STRUCTURED ASSESSMENT}

S. Lopez-Salguero $^{1}$, J.C. Ordoñez ${ }^{1}$, L. Ranieri ${ }^{1}$, M. Andrés ${ }^{1,2}$, J. Ponce $^{3}$, I. Ibero ${ }^{1} .{ }^{1}$ Rheumatology, Hospital General Universtiario Alicante;

${ }^{2}$ Departamento de Medicina Clínica, Universidad Miguel Hernández de Elche;

${ }^{3}$ Oncology, Hospital General Universtiario Alicante, Alicante, Spain

Background: Women with breast cáncer (BC) are at risk for the development of bone loss and osteoporosis (OP) mainly due to adjuvant therapies. Aromatase inhibitors (Al) therapy fully suppresses estrogen synthesis, further exacerbating the increased bone resorption and leading to an excess fracture risk. Thus, a close monitoring of bone mineral metabolism is recommended.

Objectives: The aim of the present study was to analyze bone health status and clinical characteristics of women $\mathrm{BC}$ referred by oncologist to a specialized clinic and their outcomes during follow-up.

Methods: Retrospective analysis of consecutive female patients with recent BC and low bone mineral density (BMD) referred to the osteoporosis outpatient clinic for assessment, as agreed with oncologists. A descriptive analysis of epidemiological, clinical, laboratory, imaging, and dual energy $x$-ray absorptiometry (DEXA) data is presented, both at baseline and last visit. $95 \%$ confidence intervals $(95 \% \mathrm{Cl})$ were estimated for rate of fragility fractures (FF) at baseline and during follow-up. Results: A total number of 156 female patients have been assessed up to January 2017; median aged $60.7(\mathrm{SD} \pm 10.6)$ years old, $89 \%$ postmenopausal. BC was mainly non-methastasic $(117 ; 75 \%)$, and $110(70 \%)$ patients were on aromatase inhibitors (14 on anastrozole, 89 on letrozole, and 7 on exemestane). 36 patients $(23 \%)$ were active smokers, and $17(11 \%)$ had stopped smoking. At baseline, 37 patients $(24.2 \%, 95 \mathrm{Cl} \% 20-37)$ had previous FF, mostly vertebral (19) or non-vertebral (13), and three cases had suffered from multiple FF. BMDs were at osteoporotic range at the lumbar spine (median T-score -2.6 p25/75 -2.2, -3.2) and osteopenic at both the femoral neck and hip (mediant T-score -1.9 p25/75 -1.1, -2.3). Regarding antiosteoporotic therapies, bisphosphonates were prescribed in 110 cases $(70.5 \%)$, denosumab in $24(15.3 \%)$, and raloxifene in two cases $(1.2 \%)$; in the others $(12.8 \%)$ only calcium plus vitamin D supplementation was recommended. A total of 107 patients were followed a median of 2.13 years (p25-75 1.23-3.18). During follow-up, new FF occurred in 13 patients $(12.1 \%$, $95 \% \mathrm{Cl} 6-19$ ), that were vertebral in 8 , non-vertebral in 4 , both in one case, while no hip FF were detected.

Conclusions: outcomes of a structured assessment of female patients with BC and low BMD are reported here. Despite this, $12 \%$ of cases developed a new $\mathrm{FF}$, highlighting the need for special attention to this singular, secondary form of osteoporosis.

Disclosure of Interest: None declared

DOI: 10.1136/annrheumdis-2017-eular.4928

\section{FRI0571 OSTEOPOROSIS AND BREAST CANCER: CAN FRAX-BASED RISK FACTORS ACCURATELY PREDICT FURTHER FRACTURES AT THIS SETTING?}

S. Lopez-Salguero $^{1,1}$, J.C. Ordoñez ${ }^{1}$, L. Ranieri ${ }^{1}$, M. Andrés ${ }^{1,2}$, J. Ponce ${ }^{3}$, I. Ibero ${ }^{1} .{ }^{1}$ Rheumatology, Hospital General Universtiario Alicante;

${ }^{2}$ Departamento de Medicina Clínica, Universidad Miguel Hernández de Elche;

${ }^{3}$ Oncology, Hospital General Universtiario Alicante, Alicante, Spain

Background: Women with breast cáncer (BC) are at risk for the development of bone loss and osteoporosis (OP) mainly due to adjuvant therapies, as aromatase inhibitors (Al). Thus, it would be of special interest in this group of patientes, to know at baseline wich factors can predispose to develope fragility fractures (FF) during follow-up, in order to optmize vigilance and treatment.

Objectives: The purpose of this study is to analyze wich risk factors at baseline that can predict the appereance of a new FF in women with $\mathrm{BC}$ and $\mathrm{OP}$.
Methods: Retrospective analysis of consecutive female patients with recent breast cancer (BC) and low bone mineral density (BMD) referred to the osteoporosis outpatient clinic for assessment, as agreed with oncologists. Fort he purpose of this anaylisis, only patients with follow-up data (at least six months after baseline visit) were selected. FRAX tool [1]-derived risk factors (age, BMI, DEXA, previous fracture, parent fractured hip, smoking, alcohol, glucocorticoids, rheumatoid arthritis, secondary OP) were taken as explicative variables. Student's $t$ and chi-2 tests were used to perform comparisons base don the appereance of new FF in the study period.

Results: A total number of 156 female patients have been assessed up to January 2017. Of the 107 patients in follow-up (68.5\%; median time in follow-up 2.1 years p25-75 1.2-3.2). Median age was 62.07 years old $(S D \pm 10,35)$, being $89 \%$ of them postmenopausal. $73(68,2 \%$ ) were on Al therapy (10 anastrozole, 59 letrozole and 4 exemestane). At baseline, 29 patients (27.1\%) showed a FF (15 vertebral; 10 non vertebral; 2 hip; 2 multiple fracture). Antiosteoporotic treatment was recommended in 95 patients $(88.7 \%)$. During follow-up, $13 \mathrm{FF}$ were seen $(12,1 \%$; Cl95\% 6-19); being 8 of them vertebral, 4 not vertebral, and one multiple fracture; no new hip FF was seen.

After comparison of the different risk factors according to the development of a new FF, no significant association was found (see table).

Table 1

\begin{tabular}{|c|c|c|c|}
\hline & \multicolumn{2}{|c|}{ New fragility fracture } & \multirow[t]{2}{*}{$\mathrm{p}$} \\
\hline & $\mathrm{NO}(\mathrm{n}=94)$ & YES $(n=13)$ & \\
\hline Age (years old), mean $\pm S D$ & $62.0 \pm 10.7$ & $62.1 \pm 9.9$ & 0.956 \\
\hline $\mathrm{BMI}\left(\mathrm{kg} / \mathrm{m}^{2}\right)$, mean $\pm \mathrm{SD}$ & $26.5 \pm 7.1$ & $23.1 \pm 10.0$ & 0.213 \\
\hline Follow-up duration (months) median $\pm \mathrm{SD}$ & $28.6 \pm 19,4$ & $30.6 \pm 12.8$ & 0.723 \\
\hline DEXA at lumbar spine (T-score) median $\pm S D$ & $-2.7 \pm 0,8$ & $-2.9 \pm 0.6$ & 0.256 \\
\hline $\mathrm{GFR}(\mathrm{m} / \mathrm{min}) \mathrm{mean} \pm \mathrm{SD}$ & $91.7 \pm 16.0$ & $97.2 \pm 11.2$ & 0.282 \\
\hline Menopause (\%) & 93.4 & 91.6 & 0.822 \\
\hline Previous fracture (\%) & 27.1 & 38.4 & 0.512 \\
\hline Parent fractured hip (\%) & 11.0 & 25.0 & 0.125 \\
\hline Smoking (\%) & 10.0 & 8.3 & 0.721 \\
\hline Glucocorticoids (\%) & 9.9 & 7.7 & 1.000 \\
\hline Rheumatoid arthritis (\%) & 3.2 & 0 & 1.000 \\
\hline Aromatase inhibitor (\%) & 70.9 & 69.2 & 0.897 \\
\hline Antiosteoporotic treatment (\%) & 88.4 & 92.3 & 0.676 \\
\hline
\end{tabular}

Conclusions: In this study no relationship between FRAX-based risk factors and the development of new FF in women with OP and BC was found. As new FF occurred in $12 \%$ of cases, it highlights the need for special attention to this singular, secondary form of OP.

References:

[1] Kanis JA, Johnell O, Oden A, Jonsson B, Dawson A, Dere W. Risk of hip fracture derived from relative risks: an analysis applied to the population of Sweden. Osteoporosis International 2000; 11: 120-127.

Disclosure of Interest: None declared

DOI: 10.1136/annrheumdis-2017-eular.5211

\section{FRI0572 IDENTIFYING WOMEN AND MEN AT HIGH FRACTURE RISK BY LEVERAGING THE ELECTRONIC MEDICAL RECORDS TO ESTIMATE FRAX TREATMENT THRESHOLDS}

\section{S. Amin, E. Atkinson, S. Khosla. Mayo Clinic, Rochester, United States}

Background: In the busy clinic, identifying individuals at high fracture (fx) risk who warrant intervention can be a challenge. There are several medical conditions that increase the risk for bone loss and falls which are recorded in the electronic medical record (EMR).

Objectives: We explored whether we could exploit data available in the EMR to estimate FRAX treatment thresholds to help passively identify patients who would benefit from further bone mineral density screening and management.

Methods: We studied 912 women and men, previously recruited for our bone health studies, in whom FRAX scores (with and without BMD) had been determined and comprehensive medical diagnoses were available through the medical linkage system of the Rochester Epidemiology Project. All diagnoses were categorized by the Clinical Classification Software (CCS) system whereby over 14,000 ICD-9-CM diagnoses are reduced to 568 clinically meaningful categories. If a subject had at least two diagnoses in a CCS category that were at least 30 days apart and within 5 years of their FRAX assessment, the subject was treated as having that CCS code. We used Gradient Boosting Machine (GBM) to create models that would predict the treatment thresholds for the FRAX 10-year risk for major osteoporotic (OP) fx (>20\%) and hip fx (>3\%), based on available diagnoses. Models were fit using age, sex and CCS category from $80 \%$ of the data, retaining $20 \%$ for validation.

Results: Of the $564(62 \%)$ women and $348(38 \%)$ men, the mean \pm SD age was $61 \pm 16 \mathrm{yrs}$. There were no significant differences in subject characteristics used for FRAX calculation or FRAX scores between the training and validation sets. The c-statistic for GBM models predicting treatment thresholds for FRAX, calculated with BMD, for major OP fx and hip fx were 0.95 and 0.96 , respectively, for the training set, and 0.88 and 0.94 for the validation set. Similar results were observed for FRAX scores without BMD.

Conclusions: FRAX treatment thresholds may be reasonably estimated from data available in the EMR to help identify to the clinician those at highest risk 
for fracture who would warrant further evaluation. Further work to implement and validate these findings in the EMR system would be necessary.

Disclosure of Interest: None declared

DOI: 10.1136/annrheumdis-2017-eular.6572

\section{FRI0573 OSTEOCYTES ARE INVOLVED IN THE PATHOGENESIS OF OSTEOPOROSIS IN CHRONIC CHOLESTASIS. EFFECTS OF BILIRUBIN AND BILE ACIDS ON OSTEOCYTIC CELL LINES}

S. Ruiz-Gaspà, A. Parés, A. Combalia, P. Peris, A. Monegal, N. Guañabens. Liver and Metabolic Bone Diseases Units, CIBERehd-Hospital Clínic, University of Barcelona, Barcelona, Spain

Background: Mechanisms underlying osteoporosis in chronic cholestasis are complex and poorly understood. In this setting, osteoporosis mainly results from low bone formation, related to the effects of retained substances of cholestasis, such as bilirubin and bile acids. Thus, in "in vitro" studies, unconjugated bilirubin and serum from jaundiced patients decrease osteoblast viability, differentiation and mineralization. However, the influence of cholestasis on osteocytes, the most ubiquitous cells of the skeleton, is unknown.

Objectives: The aim of this study was to analyze the direct effects of increased molecules of cholestasis, such as bilirubin (Bil) and lithocholic acid (LCA), and the potential protective effect of ursodeoxycholic acid (UDCA) on the osteocytes. Methods: MLO-Y4 and MLO-A5 osteocyte cell lines treated at different times and concentrations with Bil, LCA and UDCA were used to determine: 1) Viability: WST colorimetric method; 2) Differentiation: quantification of alkaline phosphatase (AP) activity; 3) Mineralization: Alizarin red staining quantification; and 4) Apoptosis: quantification of DNA fragmentation and caspase-3 activity.

Results: LCA $(100 \mu \mathrm{M})$ and Bil $(50 \mu \mathrm{M})$ significantly decreased viability in MLOY4 from 72 hours $(10 \%)$ and 48 hours $(11 \%)$, respectively $(\mathrm{p}<0.01)$, and Bil decreased viability $(49 \%)$ in MLO-A5 from 96 hours $(p<0.01)$. Bil decreased AP activity by $47 \%$ after 96 hours, under conditions of differentiation in MLO-Y4 $(p<0.01)$. There were no effects on AP activity in MLO-A5. After 14 days, Bil was associated with a significant mineralization decrease, as high as $87 \%$, in MLO-A5 $(\mathrm{p}<0.02)$. Moreover, Bil and LCA increased apoptosis in MLO-Y4, determined by DNA fragmentation (242\% and $119 \%$, respectively) and caspase- 3 activity (190\% and $251 \%$, respectively) $(\mathrm{p} \leq 0.01)$ after 24 hours. In contrast, UDCA $(100 \mu \mathrm{M})$ increased viability after 72 hours $(11 \%)$ and decreased the deleterious effects of LCA or Bil $(p \leq 0.02)$. UDCA increased AP activity in MLO-Y4 after 72 hours under growth conditions $(p=0.018)$, and after 24 hours under differentiation conditions $(\mathrm{p} \leq 0.01)$.

Conclusions: Bilirubin and lithocholic acid have damaging effects on osteocytic cells decreasing viability, differentiation and mineralization, and increasing apoptosis, effects that are neutralized by the UDCA. These results indicate that substances retained in cholestasis impair osteocytic functions, and therefore may be involved in the pathogenesis of osteoporosis in cholestatic diseases.

Disclosure of Interest: None declared

DOI: 10.1136/annrheumdis-2017-eular.4711

\section{FRI0574 QUALITY OF LIFE ASSESMENT IN POSTMENOPAUSAL OSTEOPOROSIS}

\section{S. Novkovic ${ }^{1}$, G. Stanojevic ${ }^{2} .{ }^{1}$ Institute of rheumatology; ${ }^{2}$ Health Center} "Zvezdara", Belgrade, Serbia

Objectives: To compair two questionaires:a specific one-Osteoporosis Quality of Life Questionnaire (OQLQ) and a general one-Short Form-36 (SF-36), in women with decreased mineral bone density (BMD) and vertebral fractures, and to estimate which one is more useful for everyday work.

Methods: Cross-section study included 50 menopausal women with osteoporosis (OP), who were assessed with the general questionaire, and all known risk factors for osteoporosis were confirmed. Osteodensitometry scan (DXA) was performed on lumbar spine, and based both on T index value and BMD the subjects were divided in two groups:first grour (women with OP, $n=25$ ), second group (women with fractures of spinal vertebra due to OP,regardless of BMD, $n=25$, and with at least 4 months since the last fracture). The quality of life was assessed by two questionaires:a specific one OQLQ- consists of 30 questions divided into 5 areas,which are evaluated from 1 to 7 (the lower the score the more sever function damage and worse quality of life) and the general questionnaire SF-36-containing 36 questions divided into 8 areas (scores from 0 to 100 , where the lower value is also a poorer quality of life).Questionnaires were converted to get two summarized scales,so as to obtain the total score for:physical (PCS) and mental functions (MCS). The two questionnaires were compared using appropriate statistical methods in SPSS.

Results: There was no significant differencev between groups regarding: mean age (first=63.3 \pm 6.0 , second=64.9 \pm 7.8 ), mean age at the start of the menopause (first $=46.8 \pm 5.3$, second $=48.2 \pm 4.2$ years), mean duration of menopause (first=16.4 \pm 6.9 , second $=17.1 \pm 6.9$ year), but there was statistically significant difference between the number of risk factors for OP (first=1.8 \pm 1.2 , second $=2.8 \pm 0.9, p<0.01)$. Statistically significant between group difference $(p<0.01)$ in mean BMD and T score (first-BMD $0.807 \pm 0.057 \mathrm{gr} / \mathrm{cm}^{2}, T$ scor $-3.12 \pm 0.49 \mathrm{SD}$, second- BMD $0.931 \pm 0.172 \mathrm{gr} / \mathrm{cm}^{2}, T$ score $\left.-2.09 \pm 1.45 \mathrm{SD}\right)$. The following DXA values were measured in the second group:8 patients-normal,6-osteopenia,11- osteoporosis, with average number of fractures 2.6 \pm 0.8 . There was statistically significant between group difference in values of PCS OQLQ questionnaire (first $=2.99,42.7 \%$, second $=2.30,32.8 \%, p<0.01$ ), as well as in values of MCS OQLQ (first $=3.18,45.4 \%$, second $=2.17,31.0 \%, p<0.01$ ). Statistically significant difference was also observed regarding values of PCS SF-36 (first $=42.2,42.2 \%$, second $=32.5,32.5 \%, p<0.01$ ), while regarding MCS SF-36 questionnaire there was no significant between group difference (first=40.2, $40.2 \%$, second $=33.3$, $33.3 \%, p>0.05)$. Mutual comparison of two questionnaires demonstrated correspondence and statistically significant decline in the quality of life in a group with fractures when it comes to PCS and MCS at OQLQ questionnaire and did not record between group difference in MCS SF-36 questionnaire.

Conclusions: It was demonstrated that the specific questionnaire provided more accurate information in all areas of quality of life,with general questionnaire failing to record between group differenc in the field of mental functions.Based on the proven value as an instrument of measurement, its simplicity,accuracy and ease of administration,OQLQ questionnaire is more appropriate for routine use in women with decreasing bone quality and fractures of vertebrae due to osteoporosis.

Disclosure of Interest: None declared

DOI: 10.1136/annrheumdis-2017-eular.3721

\section{FRI0575 SAFETY OF DENOSUMAB IN A MONOCENTRIC COHORT OF KIDNEY TRANSPLANT RECIPIENTS}

S. Doddoli, P. Lafforgue, T. Pham. Bouches Du Rhones, Aix Marseille Unversity, APHM, Marseille, Marseille, France

Background: The safety of Denosumab, a fully human monoclonal antibody against RANKL, which was developed for treatment of osteoporosis and prevention of fractures, remains unclear in kidney transplanted patients.

Objectives: Our aim was to assess its clinical and biological tolerance in this specific population.

Methods: Design: Prospective observational monocentric cohort. Inclusion criteria: kidney transplant recipient who received at least one subcutaneous injections of $60 \mathrm{mg}$ denosumab; age $\geq 18$ years

Safety assessment: the following variables were collected every 6 months: infection, reaction at the injection site, plasmatic parameters of renal function and mineral metabolism (estimated glomerular filtration rate, serum creatinine calcium, 1-25 $[\mathrm{OH}]$ vitamin D, PTH)

Results: Patients were recruited from April 2014 to September 2015. All patients received immunosuppression therapy including prednisolone $\geq 5 \mathrm{mg} / \mathrm{d}$. The main baseline characteristics of the 37 kidney transplant recipients were the following [mean]: male: $41 \%$, age: 60.5 years, BMI: 24,1 , transplantation duration: 7.1 years, osteopenic patients: $36 \%$, osteoporosis patients: $64 \%$, total lumbar spine T-score: -2.04 SD, total hip T-score: $-2.7 \mathrm{SD}$, T-score femoral neck: $0.676 \mathrm{~g} / \mathrm{cm}^{2}$, serum creatinine: $132.8 \mathrm{mmol} / \mathrm{L}$, calcium: $2.33 \mathrm{mmol} / \mathrm{L}, 1-25[\mathrm{OH}]$ vitamin $\mathrm{D}$ $93.5 \mathrm{nmol} / \mathrm{L}, \mathrm{PTH}$ 95: ng/l. All patients were prescribed vitamin D and calcium supplementation.

During the mean 12-month follow-up period, there were no unexpected adverse event $[A E]$ or severe adverse event, no graft failure and no deaths. No patient experienced fracture. Only one patient presented an infectious AE with recurrent cutaneous abscess. Renal function remained stable with no difference in serum creatinine between baseline and 12 months for the majority of the kidney transplant recipients. However, 9 recipients experienced a decrease in renal function with a mean increased in serum creatinine of $32.5 \mathrm{micromol} / \mathrm{L}$ between baseline and 12 months. Serum calcium was stable, no hypocalcaemia was observed. Among patients with normal baseline PTH, two presented hyperparathyroidism during the follow-up period. Among the 11 patients with baseline hyperparathyroidism, 7 had an increased PTH level between baseline and 12 months. None were initiated on cinacalcet.

Conclusions: Our results suggest that denosumab is safe in kidney transplant recipients. We did not observe an increase in the infection rates, nor hypocalcemia. However, several patients experienced a decrease in their renal function or an increased hyperparathyroidism.

Disclosure of Interest: None declared

DOI: 10.1136/annrheumdis-2017-eular.6899

\section{FRI0576 COMPARING TREATMENT INDICATION BY FRAX AND BMD ALONE IN RHEUMATIC PATIENTS ON LONG-TERM GLUCOCORTICOID IN HONG KONG}

S.L. Lau ${ }^{1}$, M.L. Yip ${ }^{2}$, L.-S. Tam ${ }^{1}$, K.L. Lee ${ }^{3}$ on behalf of The Hong Kong GIOP Study Group. ${ }^{1}$ Department of Medicine \& Therapeutics, The Chinese University of Hong Kong: ${ }^{2}$ Department of Medicine and Geriatrics, Kwong Wah Hospital; ${ }^{3}$ Department of Medicine, Pamela Youde Nethersole Eastern Hospital, Hong Kong, Hong Kong

Background: Bone mineral density (BMD) may underestimate fracture risk since most patients with fracture had osteopenia or normal BMD. The WHO Fracture Risk Assessment Tool (FRAX) incorporated BMD and clinical risk factors (CRF) to estimate the 10-year probability of major osteoporotic fractures (MOF) and hip fracture. FRAX has been adopted by various guidelines to assess the need for therapeutic intervention. Whether FRAX is superior to BMD in identifying high-risk patients in need of anti-osteoporotic treatment is worth exploring. 